\title{
Erratum to: Nonrenewal spike train statistics: causes and functional consequences on neural coding
}

\author{
Oscar Avila-Akerberg • Maurice J. Chacron
}

Published online: 31 March 2011

(c) Springer-Verlag 2011

\section{Erratum to: Exp Brain Res}

DOI 10.1007/s00221-011-2553-y

In the original publication of this article, Figure 1e had an incorrectly Labelled axis, and moreover the curves in Figure 5 were improperly labeled. The corrected version of both the figures can be found in following pages.

The online version of the original article can be found under doi:10.1007/s00221-011-2553-y.

O. Avila-Akerberg · M. J. Chacron ( $₫)$

Department of Physics, McGill University,

3655 Sir William Osler, Room 1137, Montreal,

QC H3G 1Y6, Canada

e-mail: maurice.chacron@mcgill.ca

\section{J. Chacron}

Department of Physiology,

McGill University, Montreal, QC, Canada 
Fig. 1 Example of nonrenewal spike train statistics. a Voltage trace from a pyramidal cell of weakly electric fish. The spike times and ISIs (I1,...I9) are also shown. It is seen that short ISIs tend to be followed by long ones and vice versa. b ISI probability distribution obtained from the same cell. c ISI return map obtained from the same cell. The slope of the best-fit line (gray) is equal to the ISI serial correlation coefficient at lag one for the spike train. d ISI serial correlation coefficients $\rho_{j}$ as a function of lag $\mathrm{j}$. Note that we have $\rho_{1}<0$. e The ISI

probability distribution obtained after randomly shuffling the ISI sequence. $f$ The ISI return map is significantly altered by the shuffling procedure. $\mathrm{g}$ ISI serial correlation coefficients qj as a function of lag $\mathrm{j}$ for the shuffled data. Note that $\rho_{j}=0$ for ${ }_{j}>0$
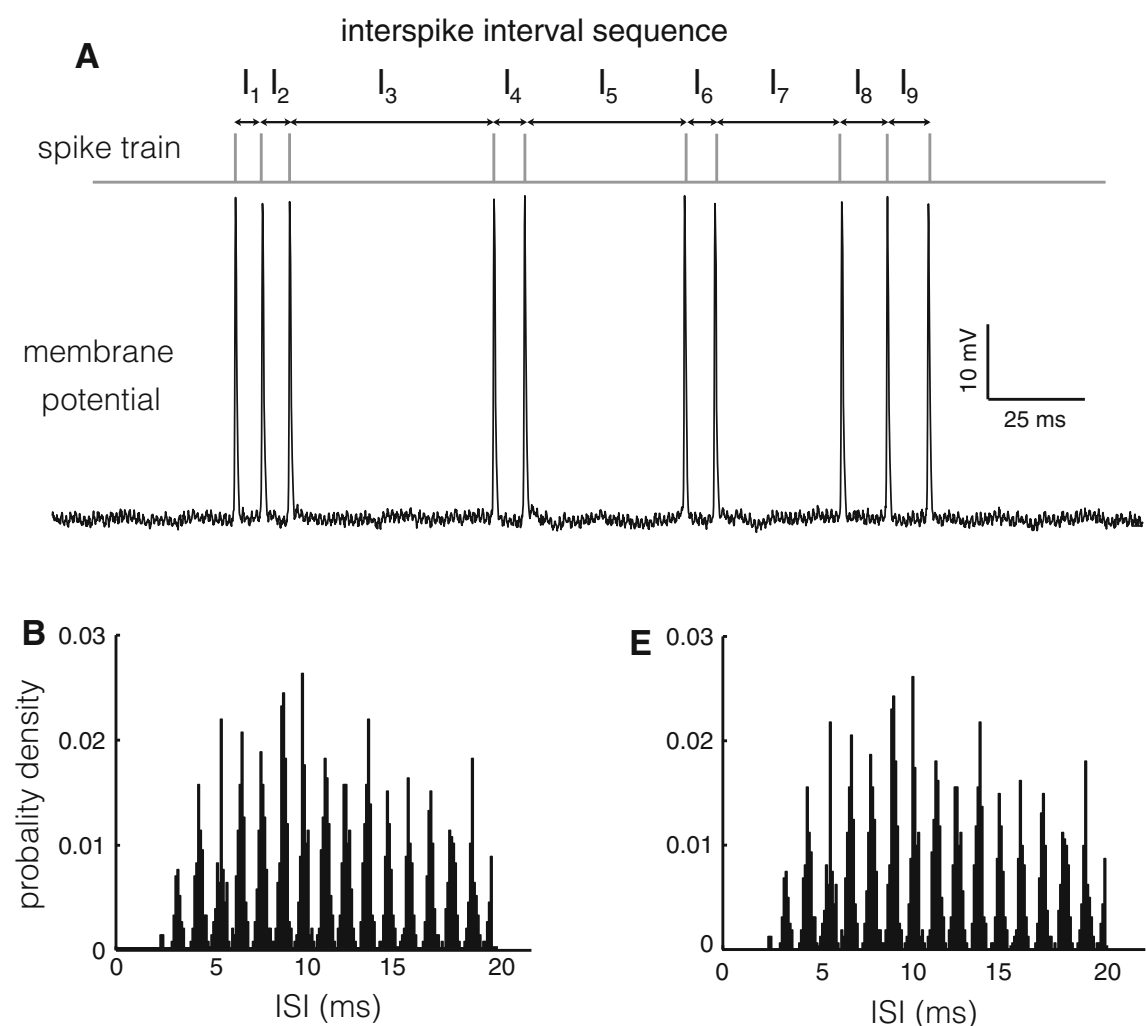

ISI (ms)

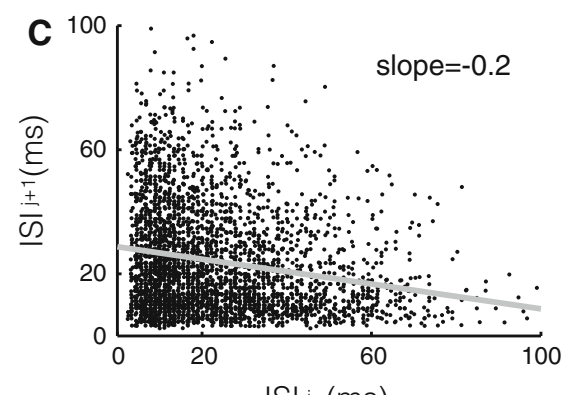

ISI j (ms)
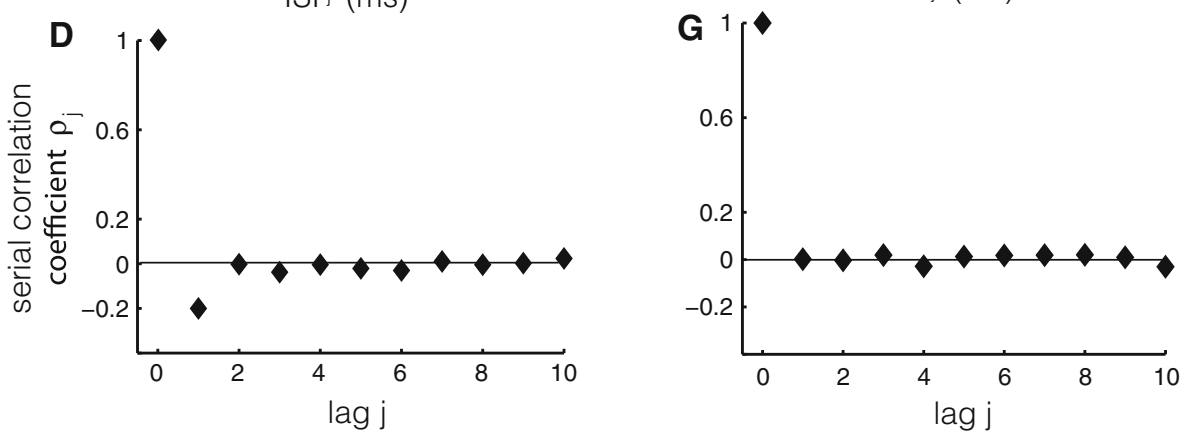


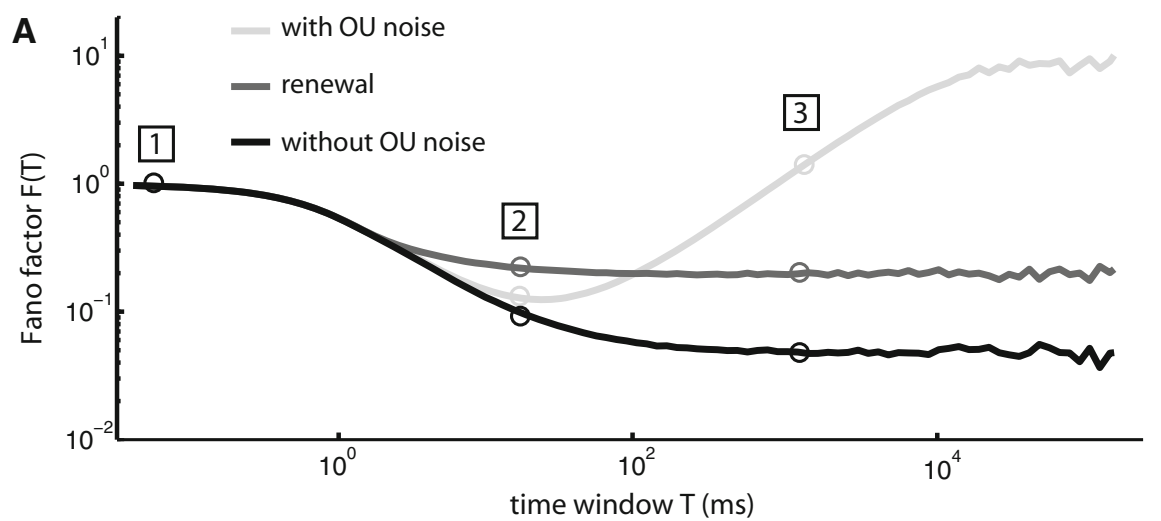

B

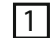

2
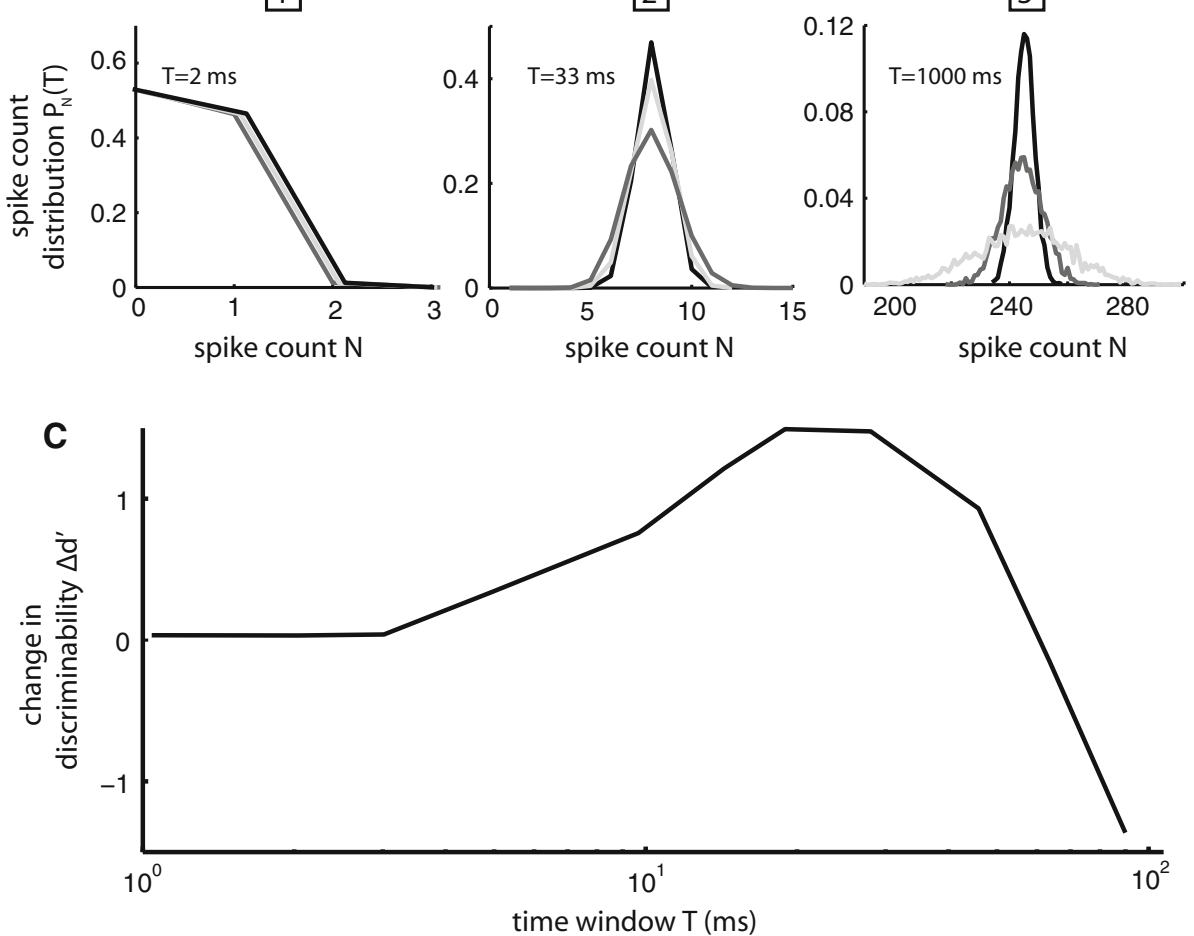

Fig. 5 Positive and negative ISI correlations act to create a minimum in spike train variability for a given time window $T$. a Fano factor $\mathrm{F}(T)$ from the LIFDT neuron model driven by a weak OrnsteinUhlenbeck (OU) noise with a long correlation time (light gray). Also shown are the Fano factor $\mathrm{F}(T)$ computed from the same model without the OU noise (black) and after shuffling the ISI sequence (dark gray). b Spike count distributions $\mathrm{PN}(T)$ obtained for all three conditions. For $T=2 \mathrm{~ms}$ (left), the distributions show almost complete overlap. However, for $T=33 \mathrm{~ms}$ (middle), the spike count distribution obtained from the LIFDT model without OU noise (black) shows the weakest variance, followed by the distribution obtained with the model with OU noise (light gray), followed by the distribution obtained after shuffling. However, for $T=1,000 \mathrm{~ms}$ (right), it is the distribution obtained from the model with OU noise (light gray) that has the highest variance. c Change in discriminability measure d' (i.e. the difference between d' computed from the model with OU noise and d' computed after shuffling the ISI sequence) as a function of the time window length $T$. It is seen that the gain in discriminability is maximal for a value of $\mathrm{T}$ for which the Fano factor $\mathrm{F}(T)$ is minimum 